\title{
8 Supplemental material
}

Table S1: Overview of the number of Avalanche Canada avalanche bulletin assessments included in the present analysis.

\begin{tabular}{|c|c|c|c|c|c|c|c|c|c|c|}
\hline Region & $\begin{array}{l}\text { Mountain } \\
\text { Range }\end{array}$ & $\begin{array}{c}2009 / \\
10\end{array}$ & $\begin{array}{l}2010 / \\
11\end{array}$ & $\begin{array}{c}2011 / \\
12\end{array}$ & $\begin{array}{l}2012 / \\
13\end{array}$ & $\begin{array}{l}2013 / \\
14\end{array}$ & $\begin{array}{l}2014 / \\
15\end{array}$ & $\begin{array}{c}2015 / \\
16\end{array}$ & $\begin{array}{c}2016 / \\
17\end{array}$ & Total \\
\hline $\begin{array}{l}\text { North Shore* } \\
\text { South Coast* }\end{array}$ & $\begin{array}{l}\text { Coast Mtn } \\
\text { Coast Mtn }\end{array}$ & $\begin{array}{c}71 \\
0\end{array}$ & $\begin{array}{c}120 \\
0\end{array}$ & $\begin{array}{c}122 \\
0\end{array}$ & $\begin{array}{c}122 \\
0\end{array}$ & $\begin{array}{c}122 \\
0\end{array}$ & $\begin{array}{c}60 \\
0\end{array}$ & $\begin{array}{c}121 \\
0\end{array}$ & $\begin{array}{c}0 \\
125^{A}\end{array}$ & $\begin{array}{l}738 \\
125\end{array}$ \\
\hline $\begin{array}{l}\text { South Coast } \\
\text { Sea-to-Sky } \\
\text { South Coast - Inland }\end{array}$ & $\begin{array}{l}\text { Coast Mtn } \\
\text { Coast Mtn } \\
\text { Coast Mtn }\end{array}$ & $\begin{array}{c}90 \\
0 \\
0\end{array}$ & $\begin{array}{c}119 \\
0 \\
0\end{array}$ & $\begin{array}{c}0 \\
123^{\text {в }} \\
123^{\text {в }}\end{array}$ & $\begin{array}{c}0 \\
122 \\
122\end{array}$ & $\begin{array}{c}0 \\
122 \\
122\end{array}$ & $\begin{array}{c}0 \\
122 \\
122\end{array}$ & $\begin{array}{c}0 \\
123 \\
123\end{array}$ & $\begin{array}{c}0 \\
127^{\mathrm{C}} \\
125\end{array}$ & $\begin{array}{l}209 \\
739 \\
737\end{array}$ \\
\hline $\begin{array}{l}\text { Northwest - BC } \\
\text { Northwest Inland } \\
\text { Northwest Coastal }\end{array}$ & $\begin{array}{l}\text { Coast Mtn } \\
\text { Coast Mtn } \\
\text { Coast Mtn }\end{array}$ & $\begin{array}{c}85 \\
0 \\
0\end{array}$ & $\begin{array}{c}118 \\
0 \\
0\end{array}$ & $\begin{array}{c}0 \\
123^{\mathrm{D}} \\
122^{\mathrm{D}}\end{array}$ & $\begin{array}{c}0 \\
122 \\
122\end{array}$ & $\begin{array}{c}0 \\
122 \\
122\end{array}$ & $\begin{array}{c}0 \\
122 \\
122\end{array}$ & $\begin{array}{c}0 \\
121 \\
123\end{array}$ & $\begin{array}{c}0 \\
123 \\
123\end{array}$ & $\begin{array}{l}203 \\
733 \\
734\end{array}$ \\
\hline Whistler Blackcomb* & Coast Mtn & 0 & 0 & 2 & 0 & 0 & 0 & 121 & 0 & 123 \\
\hline $\begin{array}{l}\text { North Columbia } \\
\text { Cariboos }\end{array}$ & $\begin{array}{l}\text { Columbia Mtn } \\
\text { Columbia Mtn }\end{array}$ & $\begin{array}{c}98 \\
0\end{array}$ & $\begin{array}{c}116 \\
0\end{array}$ & $\begin{array}{l}122^{\mathrm{E}} \\
123^{\mathrm{E}}\end{array}$ & $\begin{array}{l}122 \\
122\end{array}$ & $\begin{array}{l}122 \\
122\end{array}$ & $\begin{array}{l}122 \\
122\end{array}$ & $\begin{array}{l}123 \\
123\end{array}$ & $\begin{array}{l}130 \\
125\end{array}$ & $\begin{array}{l}955 \\
737\end{array}$ \\
\hline $\begin{array}{l}\text { South Columbia } \\
\text { Purcells }\end{array}$ & $\begin{array}{l}\text { Columbia Mtn } \\
\text { Columbia Mtn }\end{array}$ & $\begin{array}{c}87 \\
0\end{array}$ & $\begin{array}{c}116 \\
0\end{array}$ & $\begin{array}{l}123^{\mathrm{F}} \\
122^{\mathrm{F}}\end{array}$ & $\begin{array}{l}122 \\
121\end{array}$ & $\begin{array}{l}122 \\
122\end{array}$ & $\begin{array}{l}122 \\
122\end{array}$ & $\begin{array}{l}122 \\
123\end{array}$ & $\begin{array}{l}125 \\
127\end{array}$ & $\begin{array}{l}939 \\
737\end{array}$ \\
\hline Kootenay Boundary & Columbia Mtn & 85 & 120 & 123 & 122 & 122 & 121 & 123 & 129 & 945 \\
\hline $\begin{array}{l}\text { South Rockies } \\
\text { Lizard Range }\end{array}$ & $\begin{array}{l}\text { Rocky Mtn } \\
\text { Rocky Mtn }\end{array}$ & $\begin{array}{c}92 \\
0\end{array}$ & $\begin{array}{c}118 \\
0\end{array}$ & $\begin{array}{l}123^{\mathrm{G}} \\
123\end{array}$ & $\begin{array}{l}122 \\
122\end{array}$ & $\begin{array}{l}122 \\
122\end{array}$ & $\begin{array}{l}121 \\
121\end{array}$ & $\begin{array}{l}122 \\
123\end{array}$ & $\begin{array}{l}125 \\
127\end{array}$ & $\begin{array}{l}945 \\
738\end{array}$ \\
\hline Bighorn Country $-A B^{*}$ & Rocky Mtn & 6 & 17 & 0 & 0 & 0 & 0 & 0 & 0 & 23 \\
\hline North Rockies $-B C^{*}$ & Rocky Mtn & 12 & 17 & 0 & 0 & 0 & 0 & 0 & 0 & 29 \\
\hline Kananaskis Country** & Rocky Mtn & 0 & 0 & 72 & 121 & 119 & 114 & 112 & 117 & 655 \\
\hline Yukon* & $n / a$ & 0 & 0 & 41 & 0 & 36 & 18 & 13 & 0 & 108 \\
\hline & Total & 626 & 861 & 1586 & 1584 & 1619 & 1531 & 1716 & 1628 & 11152 \\
\hline
\end{tabular}

* Forecast regions excluded from analysis because of inconsistent records

5 ** Bulletins produced by Kananaskis Country, but hosted by Avalanche Canada

A Boundaries of North Shore expanded and renamed to South Coast

B Boundaries of South Coast separated into Sea-to-Sky and South Coast Inland

C Boundaries of Sea-to-Sky reduced to accommodate North Shore expansion
10

D Boundaries of Northwest - BC separated into Northwest Coastal and Northwest Inland

E Boundaries of North Columbia separated into Cariboo and North Columbia

$F$ Boundaries of South Columbia reduced to accommodate the Purcells

$G$ Boundaries of the South Rockies reduced to accommodate the Lizard Range 
Table S2: Overview of the number of Parks Canada public avalanche bulletin assessments included in the analysis.

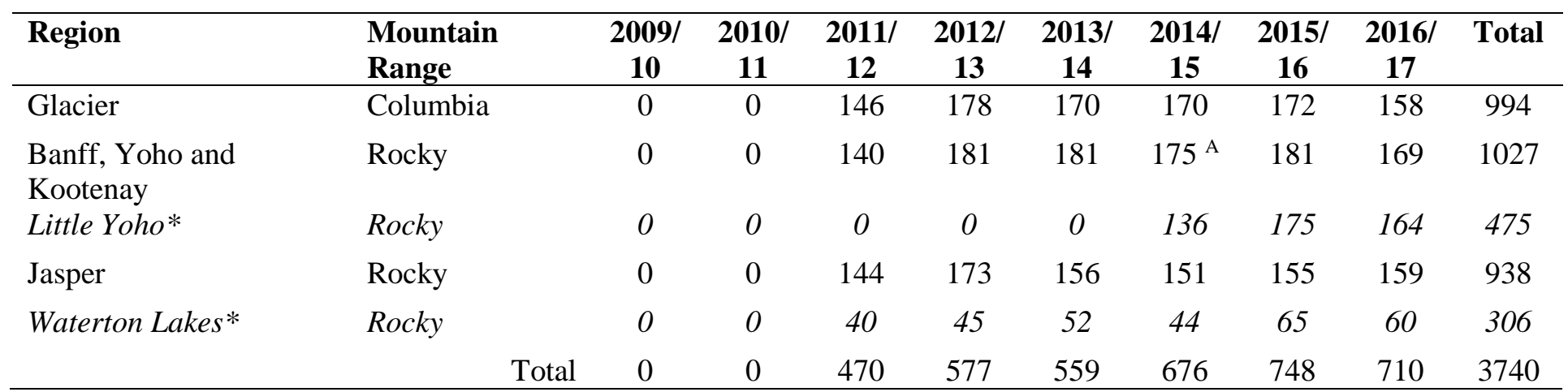

Forecast regions excluded from analysis because of inconsistent records

A Boundaries of Banff, Yoho, and Kootenay reduced to accommodate Little Yoho 
Table S3: Overview of weather data included in the present analysis and number of observations from each station during the study period.

\begin{tabular}{|c|c|c|c|c|c|c|c|c|c|c|c|c|c|}
\hline Site name & $\begin{array}{c}\text { Elevation } \\
\text { (relative } \\
\text { location) }\end{array}$ & Lat. & Long. & Source & $\begin{array}{c}\text { Forecast } \\
\text { region }\end{array}$ & $\begin{array}{c}2009 \\
/ 10\end{array}$ & $\begin{array}{c}2010 \\
/ 11\end{array}$ & $\begin{array}{c}2011 \\
/ 12\end{array}$ & $\begin{array}{c}2012 \\
/ 13\end{array}$ & $\begin{array}{c}2013 \\
/ 14\end{array}$ & $\begin{array}{c}2014 \\
/ 15\end{array}$ & $\begin{array}{c}2015 \\
/ 16\end{array}$ & $\begin{array}{c}2016 \\
/ 17\end{array}$ \\
\hline \multicolumn{14}{|c|}{ Coastal Mountains } \\
\hline $\begin{array}{l}\text { Whistler } \\
\text { Roundhouse }\end{array}$ & $\begin{array}{c}1835 \mathrm{~m} \\
(\mathrm{mtn})\end{array}$ & 50.07 & -122.95 & $\mathrm{EC}$ & Sea-to-Sky & 116 & 120 & NA & NA & 121 & 117 & 116 & 121 \\
\hline Blowdown & $\begin{array}{c}1890 \mathrm{~m} \\
\text { (mid mtn) }\end{array}$ & 50.40 & -122.47 & MOTI & $\begin{array}{l}\text { South } \\
\text { Coast } \\
\text { Inland }\end{array}$ & 121 & 121 & 122 & 121 & 119 & 121 & 122 & 121 \\
\hline Little Bear & $\begin{array}{c}1660 \mathrm{~m} \\
(\mathrm{mtn})\end{array}$ & 49.60 & -121.18 & MOTI & $\begin{array}{l}\text { South } \\
\text { Coast } \\
\text { Inland }\end{array}$ & 121 & 120 & 120 & 120 & 120 & 112 & 121 & 116 \\
\hline \multicolumn{14}{|c|}{ Columbia Mountains } \\
\hline $\begin{array}{l}\text { Sliding } \\
\text { Mountain }\end{array}$ & $\begin{array}{c}1675 \mathrm{~m} \\
(\mathrm{mtn})\end{array}$ & 53.16 & -121.48 & MOTI & Cariboos & 120 & 121 & 122 & 116 & 121 & 121 & 89 & 78 \\
\hline Sun Peaks & $\begin{array}{l}2055 \mathrm{~m} \\
(\mathrm{mtn})\end{array}$ & 50.90 & -119.92 & $\mathrm{EC}$ & $\begin{array}{l}\text { North } \\
\text { Columbia }\end{array}$ & NA & NA & 117 & 107 & 116 & 113 & 115 & 119 \\
\hline Apex & $\begin{array}{c}1750 \mathrm{~m} \\
(\mathrm{mid} \mathrm{mtn})\end{array}$ & 49.40 & -119.90 & MOTI & $\begin{array}{l}\text { Kootenay } \\
\text { Boundary }\end{array}$ & 119 & 121 & 122 & 121 & 121 & 121 & 121 & 121 \\
\hline $\begin{array}{l}\text { London } \\
\text { Ridge }\end{array}$ & $\begin{array}{l}2070 \mathrm{~m} \\
(\mathrm{mtn})\end{array}$ & 50.04 & -117.24 & MOTI & $\begin{array}{c}\text { South } \\
\text { Columbia }\end{array}$ & 119 & 119 & 122 & NA & 121 & 115 & 119 & 117 \\
\hline Whitewater & $\begin{array}{c}1950 \mathrm{~m} \\
(\mathrm{mtn})\end{array}$ & 49.44 & -117.15 & InfoEx & $\begin{array}{l}\text { Kootenay } \\
\text { Boundary }\end{array}$ & NA & 104 & 112 & 114 & 108 & 103 & NA & 113 \\
\hline $\begin{array}{l}\text { Kootenay } \\
\text { Pass }\end{array}$ & $\begin{array}{c}1780 \mathrm{~m} \\
(\text { mid mtn) }\end{array}$ & 49.06 & -117.04 & MOTI & $\begin{array}{l}\text { Kootenay } \\
\text { Boundary }\end{array}$ & 120 & 121 & NA & NA & 120 & 117 & NA & 107 \\
\hline \multicolumn{14}{|c|}{ Rocky Mountains } \\
\hline $\begin{array}{l}\text { Chatter } \\
\text { Creek }\end{array}$ & $\begin{array}{l}1615 \mathrm{~m} \\
\text { (valley) }\end{array}$ & 51.86 & -117.60 & InfoEx & - & 112 & NA & 108 & 111 & 106 & NA & NA & 113 \\
\hline Panorama & $\begin{array}{c}2356 \mathrm{~m} \\
(\mathrm{mtn})\end{array}$ & 50.43 & -116.20 & InfoEx & Purcells & 114 & 118 & 119 & 113 & 118 & 119 & 121 & 121 \\
\hline Lake Louise & $\begin{array}{c}2200 \mathrm{~m} \\
(\mathrm{mtn})\end{array}$ & 51.46 & -116.12 & InfoEx & $\begin{array}{l}\text { Banff, } \\
\text { Kootenay } \\
\text { Yoho }\end{array}$ & 79 & 77 & NA & 112 & 99 & 112 & 119 & 119 \\
\hline Kananaskis & $\begin{array}{l}1890 \mathrm{~m} \\
\text { (valley) }\end{array}$ & 50.79 & -115.31 & InfoEx & $\begin{array}{l}\text { Kananaskis } \\
\text { Country }\end{array}$ & 84 & 101 & 111 & 109 & 89 & 110 & 109 & 112 \\
\hline
\end{tabular}


MOTI Ministry of Transportation and Infrastructure

EC Environment Canada

NA Forecast regions excluded from analysis because of inconsistent records

mid mtn Mid-mountain

$5 \mathrm{mtn} \quad$ Mountain ridgetop 